\title{
Usefulness of operative planning based on 3-dimensional CT cholangiography for biliary malignancies.
}

\section{$\operatorname{AUTHOR}(\mathrm{S})$ :}

Okuda, Yukihiro; Taura, Kojiro; Seo, Satoru; Yasuchika, Kentaro; Nitta, Takashi; Ogawa, Kohei; Hatano, Etsuro; Uemoto, Shinji

\section{CITATION:}

Okuda, Yukihiro ... [et al]. Usefulness of operative planning based on 3-dimensional CT cholangiography for biliary malignancies.. Surgery 2015, 158(5): 1261-1271

\section{ISSUE DATE:}

2015-11

URL:

http://hdl.handle.net/2433/203042

\section{RIGHT:}

(c) 2015. This manuscript version is made available under the CC-BY-NC-ND 4.0 license

http://creativecommons.org/licenses/by-nc-nd/4.0/; The full-text file will be made open to the public on 1 November 2016 in accordance with publisher's 'Terms and Conditions for Self-Archiving'.; This is not the published version. Please cite only the published version.; この論文は出版社版でありません。引用の際には出版社版をご確認ご利用ください。 


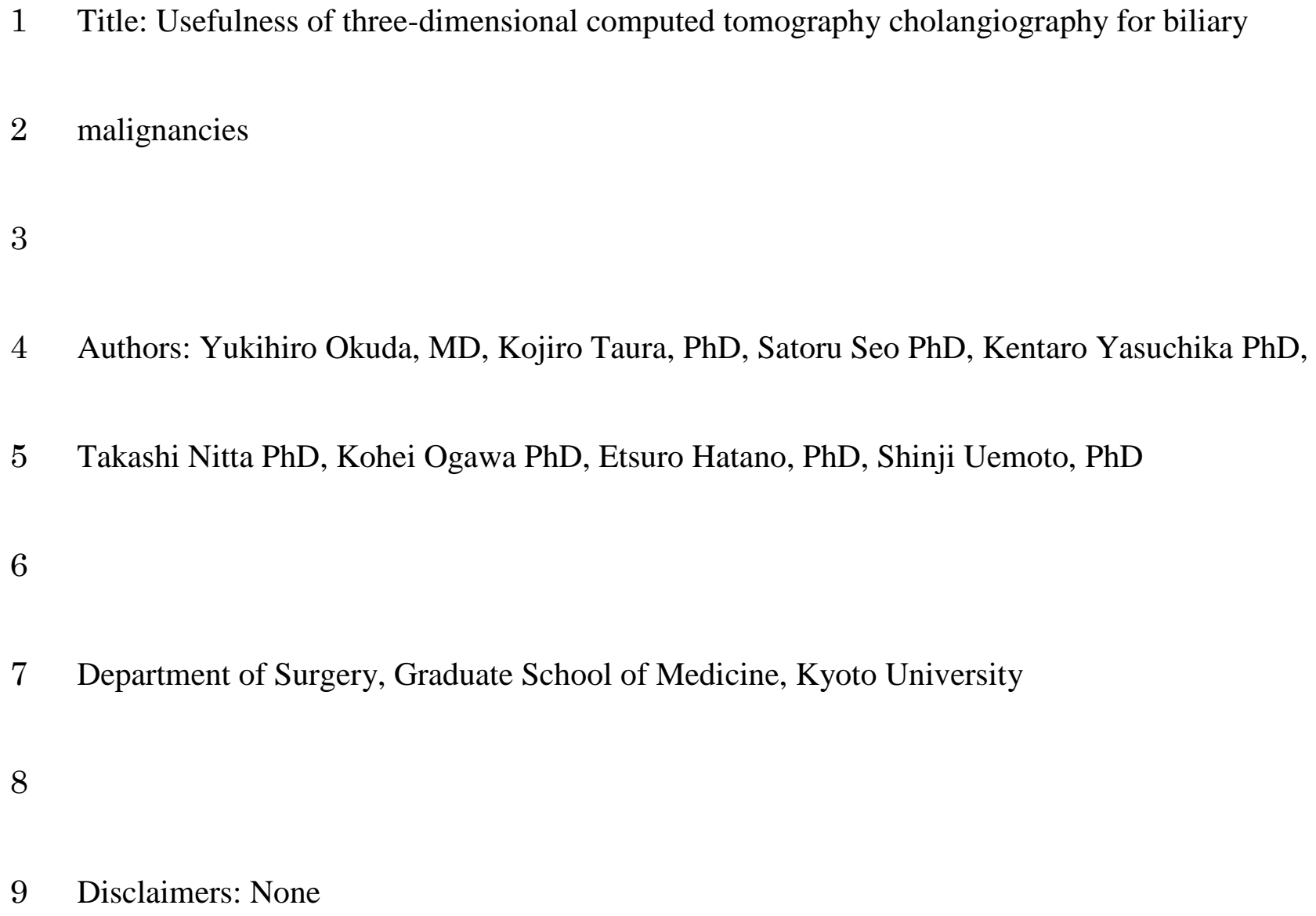




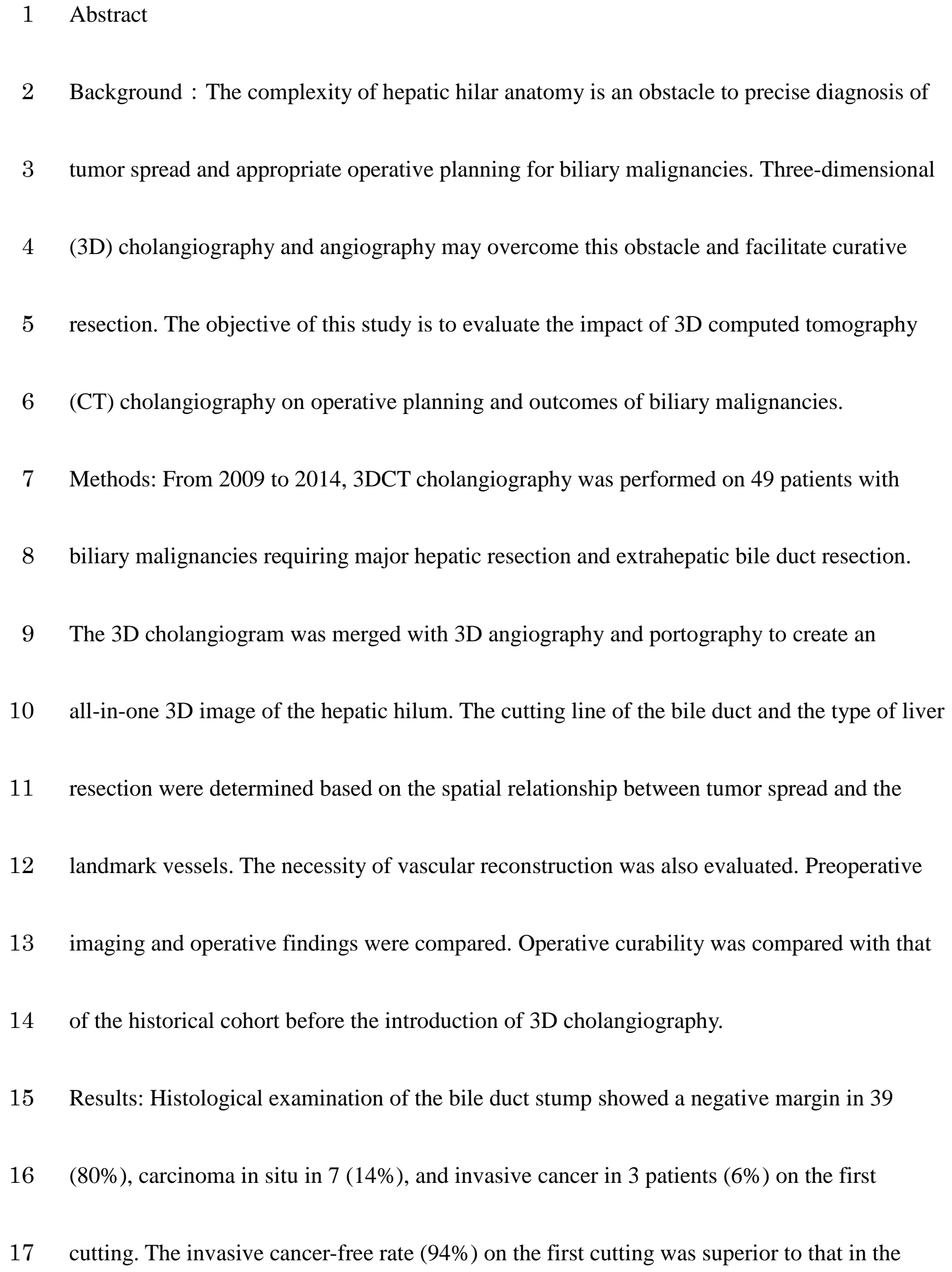


11 historical cohort $(80 \%)(\mathrm{p}=0.02)$. The necessity for portal/arterial reconstruction was

2

3

4

5

6

7

3 Conclusion: 3D cholangiography provides accurate information about hilar anatomy and

4 plays a role in facilitating adequate operative planning. 


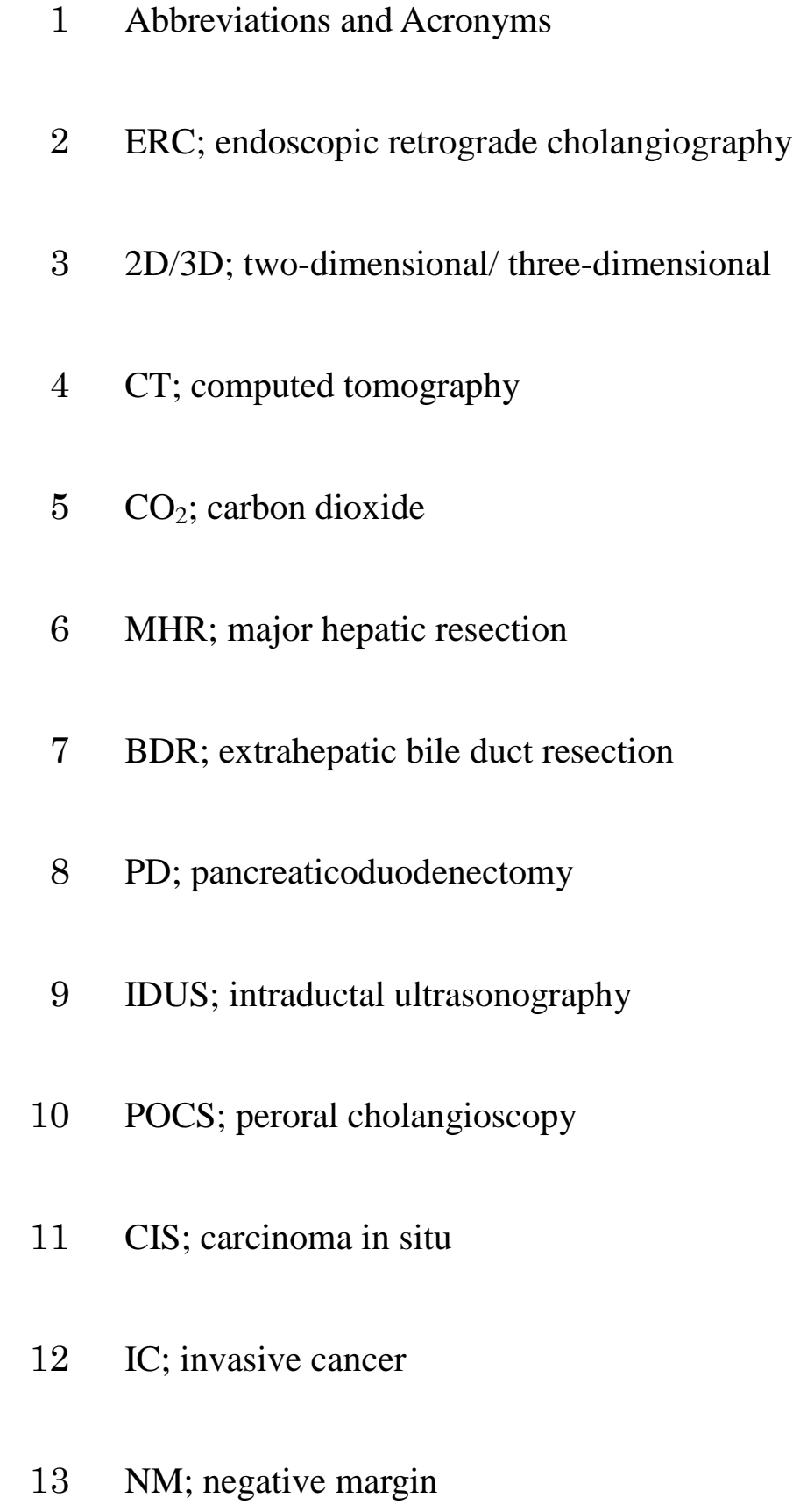




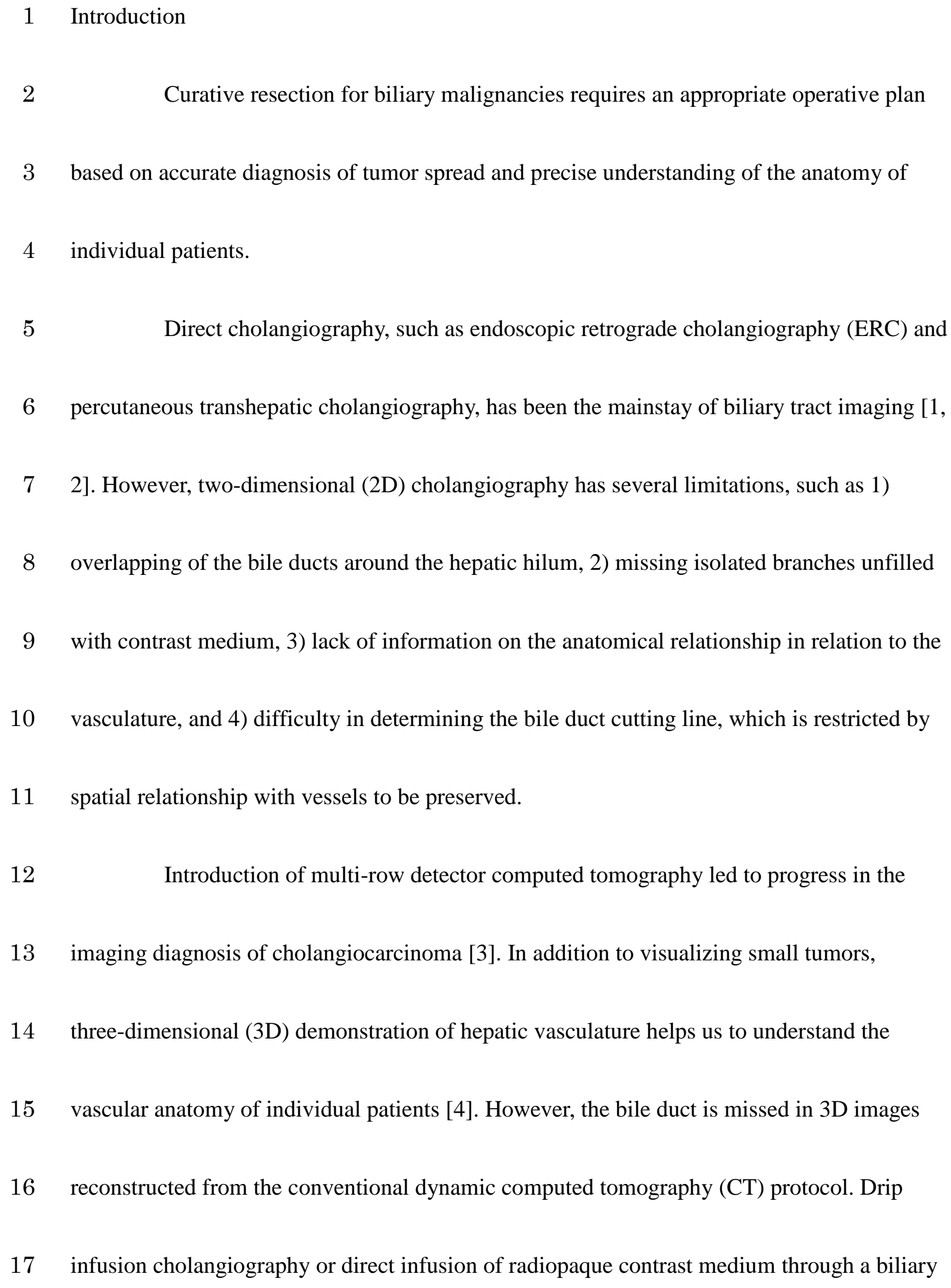

6 percutaneous transhepatic cholangiography, has been the mainstay of biliary tract imaging [1,

7 2]. However, two-dimensional (2D) cholangiography has several limitations, such as 1)

8 overlapping of the bile ducts around the hepatic hilum, 2) missing isolated branches unfilled

9 with contrast medium, 3) lack of information on the anatomical relationship in relation to the 10 vasculature, and 4) difficulty in determining the bile duct cutting line, which is restricted by 11 spatial relationship with vessels to be preserved.

Introduction of multi-row detector computed tomography led to progress in the

13 imaging diagnosis of cholangiocarcinoma [3]. In addition to visualizing small tumors,

14 three-dimensional (3D) demonstration of hepatic vasculature helps us to understand the

15 vascular anatomy of individual patients [4]. However, the bile duct is missed in 3D images

16 reconstructed from the conventional dynamic computed tomography (CT) protocol. Drip

17 infusion cholangiography or direct infusion of radiopaque contrast medium through a biliary 
1 drainage tube visualizes the bile ducts as radiopaque objects and enables its $3 \mathrm{D}$ reconstruction

2 [5]. However, with these methods, it is difficult to separate the bile ducts from the arteries,

3 since the two structures are anatomically adjacent and contrasted with similar radiopacity, and

4 they are therefore not amenable for creation of an "all-in-one" (bile ducts, arteries, and portal

5 veins) 3D fusion image. Recently, Sugimoto et al. reported the utility of carbon dioxide $\left(\mathrm{CO}_{2}\right)$

6 as a radiolucent contrast material for the biliary system [6]. The radiolucent characteristics of

$7 \quad \mathrm{CO}_{2}$ facilitated all-in-one $3 \mathrm{D}$ reconstruction by visualizing the bile ducts as radiolucent

8 objects.

In 2009, we introduced 3DCT cholangiogram, as well as all-in-one 3D imaging, for

10 precise understanding of the hepatic hilum anatomy, and have used it for operative planning

11 for biliary malignancies. In the present study, our experience with 3DCT cholangiography is

12 reviewed, with the aim of evaluating its usefulness in patients with biliary malignancies.

14 Methods

15 Study population

The study population is summarized in figure 1. We introduced 3DCT

17 cholangiography as a routine preoperative examination on October 2009. Since that time, all 
1 the patients with biliary malignancies who were candidates for partial liver resection with

2 concomitant extrahepatic bile duct resection (cholangiocarcinoma and gallbladder carcinoma

3 with hepatic hilum invasion) were prospectively enrolled. Eighty-seven patients underwent

4 3DCT cholangiography. Eight patients underwent a second examination after replacement or

5 addition of biliary drainage tubes in the contralateral side because of the need for

6 reconsideration of the operative procedures, and, as a result, 96 examinations were performed

7 in total. Of these 87 patients, 49 underwent major hepatic resection (MHR) with extrahepatic

8 bile duct resection (BDR) or MHR with pancreaticoduodenectomy (PD) (perihilar

9 cholangiocarcinoma in 25 patients, extrahepatic cholangiocarcinoma extending to the

10 perihilar region in 5 patients, intrahepatic cholangiocarcinoma with perihilar invasion in 14

11 patients, or gallbladder cancer with periductal invasion in 4 patients), and they were the main

12 participants in this study. One patient had both extrahepatic cholangiocarcinoma and

13 intrahepatic cholangiocarcinoma. Written informed consent was obtained from all patients

14 before 3D cholangiography. The protocol for this study was approved by the institutional

15 ethics committee. This study was registered with the University hospital Medical Information

16 Network (unique trial number: UMIN R000018554). 
3DCT cholangiography

2 The protocols for 3DCT cholangiography are summarized in figure 2. Of the 96

3 examinations, the contrast medium for the bile duct was $\mathrm{CO}_{2}$ in 81 and iodine $(30 \mathrm{mg} / \mathrm{ml})$ in

4 15. The choice of contrast medium was based on the severity of the bile duct stricture; $\mathrm{CO}_{2}$

5 for cases with severe stricture and iodine for those with mild or no stricture. $\mathrm{CO}_{2}$ or iodine

6 was injected via a biliary drainage tube. The volume of injected $\mathrm{CO}_{2}$ was $10-120 \mathrm{~mL}$. It was

7 adjusted depending on the severity of the stricture and the resistance perceived by the

8 examiners during the injection. After confirmation of sufficient visualization of the bile ducts

9 by a precontrast scan, a multiphase dynamic CT scan was performed in the usual manner.

In cases in which iodine was used for the bile ducts, a multiphase dynamic CT

11 scan was performed, accompanied by an additional scan after the diluted iodine $(10-35 \mathrm{~mL})$

12 was injected via the biliary tube.

The bile ducts, the artery, and the portal vein were extracted from the CT scan, and

14 3D images were constructed using image analysis programs (Virtual Place [AZE] [from

15 October 2009 to May 2012; 62 examinations], Ziostation [Ziosoft] [from June 2012 to March

$162013 ; 14$ examinations], or Synapse Vincent [Fujifilm medicals] [from April 2013 to March

$172014 ; 20$ examinations]). In $\mathrm{CO}_{2}$ cholangiography, the color mapping for CT colonoscopy or 
1 lung volumetry was applied for bile duct 3D construction. The maximum order of the

2 visualized biliary branch on 3D cholangiography was evaluated in the liver remnant, where,

3 in most cases, the biliary drainage tube was inserted.

4 Hematological examination was performed on the following day in all patients to

5 evaluate the adverse effects induced by 3D cholangiography.

$7 \quad$ Other preoperative evaluations In all 49 patients who underwent MHR+BDR or MHR+PD, 2D cholangiography

9 was also performed via a biliary tube. Intraductal ultrasonography (IDUS), endoscopic

10 ultrasonography, and peroral cholangioscopy (POCS) were performed in 30, 17, and 9 cases,

11 respectively. Tumor biopsy under ERC or POCS was performed in 36 patients. Step biopsy to

12 determine the cutting line of the bile duct was done in 36 patients.

13

14 Operative planning and procedures using 3D imaging

15 Longitudinal spread was evaluated based on the combination of 2D/3D

16 cholangiography, axial and coronal CT scan images, IDUS, POCS, and step biopsy. Tumor

17 extension was mapped onto the 3D bile duct image. The 3D bile duct image was merged with 
$13 \mathrm{D}$ images of the artery and the portal vein to generate an "all-in-one" 3D image of the

2 hepatic hilum. The anatomical relationships between tumor spread and the landmarks that

3 restrict the cutting line of the bile ducts in each operative procedure were evaluated. The

4 landmark was the umbilical portion for right side hepatectomy (U point) and the anterior

5 branch of the right portal vein for left side hepatectomy (A point) (Figure 3, please see Video

61 as well). The peripheral limit of the bile duct cutting line was the left border of the U point

7 in right trisectionectomy (Figure $3 \mathrm{I}$ ), the right border of the $\mathrm{U}$ point in right

8 hemihepatectomy (Figure 3 II ), the left border of the A point in left hemihepatectomy

9 (Figure $3 \mathrm{III}$ ), and the right border of the A point in left trisectionectomy (Figure 3IV). When

10 tumor spread went beyond the left border of the $\mathrm{U}$ point or the right border of the A point,

11 curative resection was judged impossible. When tumor spread was within the aforementioned

12 limit, the possible operative procedures were listed, and the most appropriate procedure was

13 selected based on the estimated surgical margin and the functional reserve of the liver

14 remnant with each procedure. If the liver remnant is less than $40 \%$ in patients with obstructive

15 jaundice or less than $30 \%$, preoperative portal vein embolization was performed to promote

16 hypertrophy of the remnant. The expected number of bile duct orifices on the stump of the

17 bile duct was counted based on 3D cholangiography and compared with the actual number 
1 that appeared on the stump during surgery to evaluate the agreement of the predicted and the

2 actual cutting line of the bile duct.

3 The necessity for reconstruction of the artery or the portal vein was first assessed by

$43 \mathrm{D}$ imaging. If the vessels that needed to be preserved were close to the tumor, tumor

5 involvement was evaluated on the 2DCT images. Absence of fat density around the vessel

6 was regarded as a sign of vascular invasion, and reconstruction was planned using the 3D

7 image. Figure 4 shows a case of intrahepatic cholangiocarcinoma in the left lobe that

8 necessitated both arterial and portal vein reconstruction. The 3D image shows the right

9 hepatic artery and the bifurcation of the portal vein were involved by the tumor (Figure 4a).

10 The planning of vascular reconstruction was done with the 3D image, and it was properly

11 carried out (Figure 4b, please see Video 2 as well).

13 Histological evaluation

14 Histological involvement of cancer cells on the proximal bile duct stump was evaluated by

15 intra-operative frozen section examination in all cases. When carcinoma in situ (CIS) or

16 invasive cancer (IC) was observed, additional resection of the bile duct with or without

17 parenchymal resection was considered. Histological involvement of the proximal bile duct 


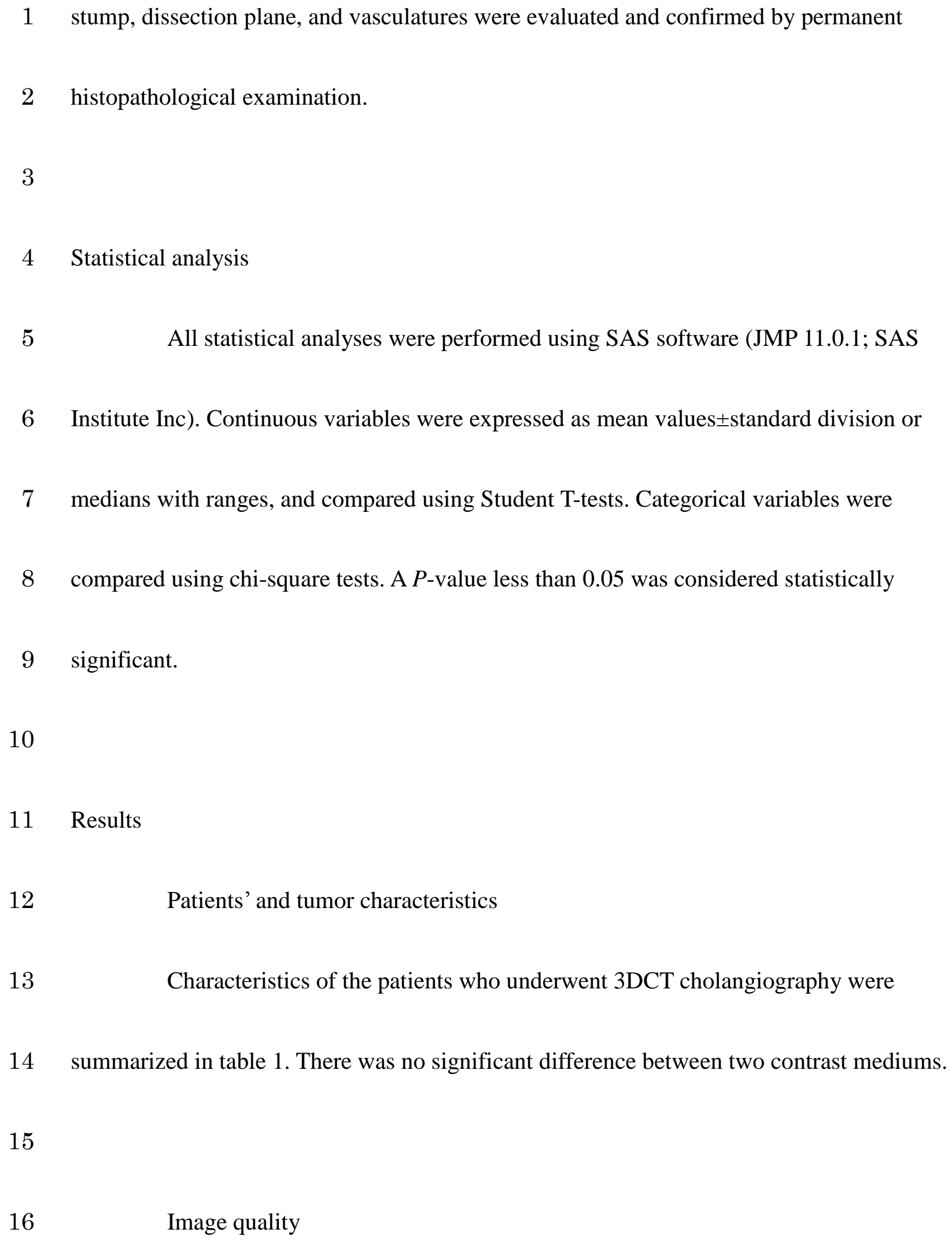


In 96 examinations, the maximum order of the visualized biliary branch on 3D

2 cholangiography was the second-order in 1 , the third-order in 8 , the fourth-order in 17 , the

3 fifth-order in 48 , and the sixth-order in 22 examinations. The median was the fifth-order, and

4 there was no significant difference between $\mathrm{CO}_{2}$ and radiopaque contrast media examinations.

5

6

All patients tolerated CT cholangiography without serious complications. Three

8 patients had mild elevations of liver enzymes. One patient undergoing $\mathrm{CO}_{2}$ cholangiography

9 developed cholangitis requiring antibiotic therapy.

10

11

13 respectively. The type of liver resection was right hemihepatectomy in 22 patients (45\%), left

14 hemihepatectomy in 13 patients (27\%), right trisectionectomy in 4 patients (8\%), and left

15 trisectionectomy in 10 patients (20\%). The median operative time was 702 minutes (range:

16456 to 1725 ), with median blood loss of $1420 \mathrm{~g}$ (range: 310 to 21736). There were no

17 operative deaths. 


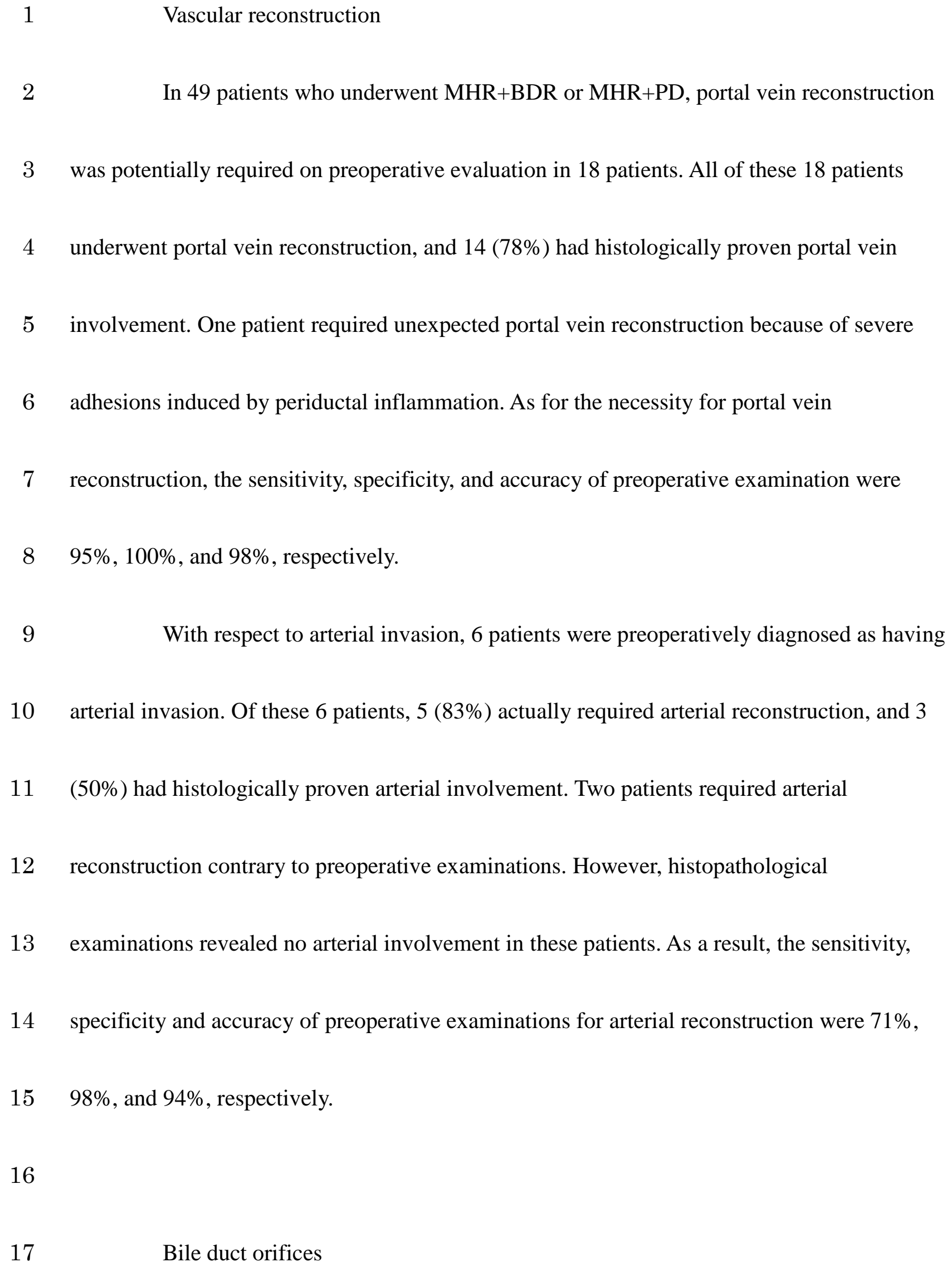


In the 49 patients, the expected number of bile duct orifices was 1 to 4 (median, 2),

2 which was in agreement with the actual number in all patients $(n=49,100 \%)$.

3

$4 \quad$ Surgical radicality

$5 \quad$ Histological involvement of cancer cells on the proximal bile duct stump was

6 evaluated in the 49 patients. A negative margin (NM) was obtained in 39 patients (80\%),

7 whereas CIS and IC were observed in 7 patients (14\%) and 3 patients (6\%), respectively, on

8 the first cutting. Additional resection of the bile duct was performed in 8 patients (5 patients

9 with CIS and 3 patients with IC), and NM was obtained in 3 patients (2 patients with CIS and

101 patient with IC). In 2 patients, additional resection was abandoned. Eventually, histological

11 evaluation of the bile duct stump was NM in 42 patients (86\%), CIS in 5 patients (10\%), and

12 IC in 2 patients (4\%). Results of histological examination on the proximal bile duct stump

13 were summarized in figure 5. R0 resection was obtained in all 42 patients with NM on the

14 bile duct stump.R0 and R1 resection rate was $86 \%$ and $14 \%$, respectively.

16 Comparison of operative curability with historical controls 
To explore the impact of preoperative 3D evaluation on operative planning and

2 outcomes, our cases who underwent MHR+BDR or MHR+PD before the introduction of 3D

3 cholangiography (from January 2004 to August 2009: n=69) were reviewed, and patients'

4 characteristics, tumor factors, and operative outcomes were compared between the present

5 series and the historical cohort (Table 2). In the historical control group, the type of liver

6 resection was right hemihepatectomy in 23 patients (33\%), left hemihepatectomy in 22

7 patients (32\%), right trisectionectomy in 14 patients (20\%), and left trisectionectomy in 10

8 patients (14\%). A non-significant downward trend in right trisectionectomy was evident after

9 the introduction of 3D cholangiography (20\% vs. $8 \%$ ).

$\mathrm{MHR}+\mathrm{PD}$, portal vein reconstruction, and arterial reconstruction were performed in

117 patients (10\%) each. A significant difference was observed in the ratio of patients

12 undergoing portal vein reconstruction $(\mathrm{p}=0.0002)$.

Histopathological evaluation of the bile duct stump on the first cutting was NM in

1451 patients (74\%), CIS in 4 patients (6\%), and IC in 14 patients (20\%). Additional resection

15 was performed in 15 patients (3 patients with CIS and 12 patients with IC), and NM was

16 obtained in 12 patients. One patient with IC on the first cutting had CIS after the additional

17 resection. Eventually, histological evaluation of the bile duct stump was NM in 61 patients 
1 (88\%), CIS in 3 patients (4\%), and IC in 5 patients (7\%). The invasive cancer-free rate on the

2 first cutting was significantly better in the present series ( $\mathrm{p}=0.02)$, although the difference

3 diminished after additional resections. In the historical control patients who obtained NM of

4 the bile duct stump, thirteen patients resulted in R1 resection because of positive margin of

5 the dissection plane in 9 patients, IC and CIS of bile duct stump by permanent pathological

6 examination in 1 patient, positive margin of portal vein cut end in one patient and distant

7 lymph node metastasis in 1 patient. As a result, $\mathrm{R} 0$ resection was achieved in 48 patients

8 (70\%). Although not statistically different, there was upward trend in the rate of R0 resection

9 in the present series ( $70 \%$ vs. $86 \%)$.

10

11

13 3D images. Figure 6a shows an infraportal bile duct branch of the Spiegel lobe, which

14 hampers en bloc resection during right hemihepatectomy with caudate lobectomy unless the

15 portal vein is transected and anastomosed (please see Video 3a as well). Figure $6 \mathrm{~b}$ shows an

16 infraportal bile duct branch of segment 3 (please see Video $3 b$ as well). Figure $6 c$ shows an

17 anatomical variation of the right posterior branch of the artery, in which A6 runs infraportally, 
1 whereas A7 runs supraportally (please see Video $3 \mathrm{c}$ as well). This variation is potentially

2 dangerous during left trisectionectomy, since, without recognizing this variation, A7 may be

3 mistaken for the right anterior branch and be transected. Figure $6 \mathrm{~d}$ shows an extremely rare

4 but significant anatomical variation for right trisectionectomy. A2, A3, and A4 arose

5 independently, and A2 ran medially and cranially to the left portal vein and was involved by

6 the tumor (please see Video 3d as well). Without knowing this variation, A2 may be mistaken

7 for A4, and the artery may be transected proximal to the bifurcation of A2.

9 Discussion

For biliary tract malignancies, curative resection is essential for improving

11 long-term survival, but the curative resection rate remains unsatisfactory in many reports [1,

12 7-13]. Obstacles to curative resection are not only difficulty in making an accurate diagnosis

13 of tumor spread, but also difficulty in the choice of an appropriate operative procedure due to

14 the complicated anatomy of the hepatic hilum. We introduced 3DCT cholangiography to

15 facilitate the proper choice of operative procedure based on a precise understanding of the

16 hilar anatomy of individual patients. In this study, our series was reviewed, and its impact on

17 outcomes was evaluated. 
The multidirectional view of 3D cholangiography tells us the appropriate angle on

2 which the diagnostically important portion of the bile duct is evaluable without overlapping,

3 which in turn helps us perform complete $2 \mathrm{D}$ cholangiography using $3 \mathrm{D}$ cholangiography as a

4 reference image. In addition, IDUS, POCS, and histological assessment of bile duct biopsy

5 specimens are now practical for precise diagnosis of longitudinal tumor spread $[1,2]$.

6 Projection of the examinations onto 3D cholangiography creates a 3D map of tumor spread,

$7 \quad$ which maximizes the usefulness of these fine examinations. MRCP can noninvasively reconstruct 3D imaging of the biliary tract, which is likely

9 to be considered a competing imaging modality to 3DCT cholangiography $[14,15]$. An

10 advantage of 3DCT cholangiography over MRCP is that it can provide all-in-one 3D fusion

11 images of all important structures in the hepatic hilum [16]. The fusion image makes it

12 possible to evaluate tumor spread in relation to anatomical landmarks such as the $U$ point and

13 A point. This is particularly important for planning operative procedures, since the bile duct

14 cutting line is restricted by these landmarks. Agreement of the number of the orifices

15 appearing on the bile duct stump suggests that 3D fusion images correctly predict the actual 16 cutting line of the bile duct. The improvement in the rate of negative ductal margin on first 
1 cutting after the introduction of 3DCT cholangiography may be partly attributable to selection

2 of the appropriate operative procedure.

3 Another benefit of 3D fusion images is recognition of anatomical variations.

4 Variations described in figure 6 are easily missed with 2D images or separated 3D images, but

5 they are readily recognized with all-in-one 3D fusion images. Preoperative recognition of

6 these variations is vital for performing operations smoothly and avoiding serious

7 complications.

Vascular invasion is frequent in biliary malignancies and is another important factor

9 affecting operative procedures [17]. With all-in-one fusion images, surgeons can easily

10 recognize vessels to be preserved in the planned operations and the distance between the

11 vessels and the tumor. The 3D images objectively demonstrate suspicious portions of vascular

12 invasion and prepare us to be ready for reconstruction during the operation. Indeed, the

13 necessity for vascular reconstruction was predictable in all but two cases in the present series.

14 In addition, the all-in-one 3D images provide planning of vascular reconstruction that is easily

15 understandable even for less experienced surgeons.

16 Endo has already reported the usefulness of 3DCT cholangiography [9]. The

17 uniqueness of the present study was that $\mathrm{CO}_{2}$ was used preferentially as the contrast material. 
1 The reason for preferential use of $\mathrm{CO}_{2}$ is that $\mathrm{CO}_{2}$ behaves as a negative contrast medium. A

2 negatively-enhanced bile duct can be easily separated from positively-enhanced vessels,

3 which enables 3D reconstruction of both the hepatic artery and the bile duct on the basis of

4 the arterial phase and, thereby, avoids slice gaps between multiple phases caused by

5 inconsistent breath-holding. Another advantage of $\mathrm{CO}_{2}$ over a radiopaque agent may be the

6 low incidence of cholangitis. Only one patient in the present series and no patients in the

7 previous study by Sugimoto et al. suffered from cholangitis [6]. These incidences of

8 cholangitis were relatively lower than that of previous reports in which radiopaque agent were

9 used as contrast medium [18, 19]. The reason for the low incidence of cholangitis may be

10 because $\mathrm{CO}_{2}$ leaks even through severe strictures, which prevents excessive elevation of

11 intraductal pressure during injection.

However, 3DCT cholangiography has several limitations. First, 3DCT

13 cholangiography is not an alternative to other diagnostic examinations such as IDUS, POCS,

14 and 2D cholangiography. The resolution of 3D images is restricted by, and therefore cannot be

15 superior to, that of the original CT images. Sensitivity of 3D cholangiography for subtle

16 changes or irregularities on the bile duct mucosa should not be as high as that of 2D

17 cholangiography. We would like to emphasize that 3DCT cholangiography is useful not for 
1 fine diagnosis of tumor extension but as a reference image for the other diagnostic modalities

2 and appropriate operative planning based on precise understanding of biliary anatomy. Second,

3 the quality of images is somewhat dependent on experience, because CT cholangiography is

4 not a real-time fluoroscopic examination. The choice and the volume of contrast material

5 (either $\mathrm{CO}_{2}$ or radiopaque agent) to be injected should be adjusted according to the severity of

6 the stricture.

In conclusion, 3D cholangiography and all-in-one 3D fusion images of the hepatic

8 hilum provide accurate information about hilar anatomy and play a role in adequate operative

9 planning. They also facilitate precise implementation of the planned surgery. We hope this

10 technique will become widespread and improve the prognosis of patients with biliary

11 malignancies by refining the complicated operations required for these intractable diseases. 


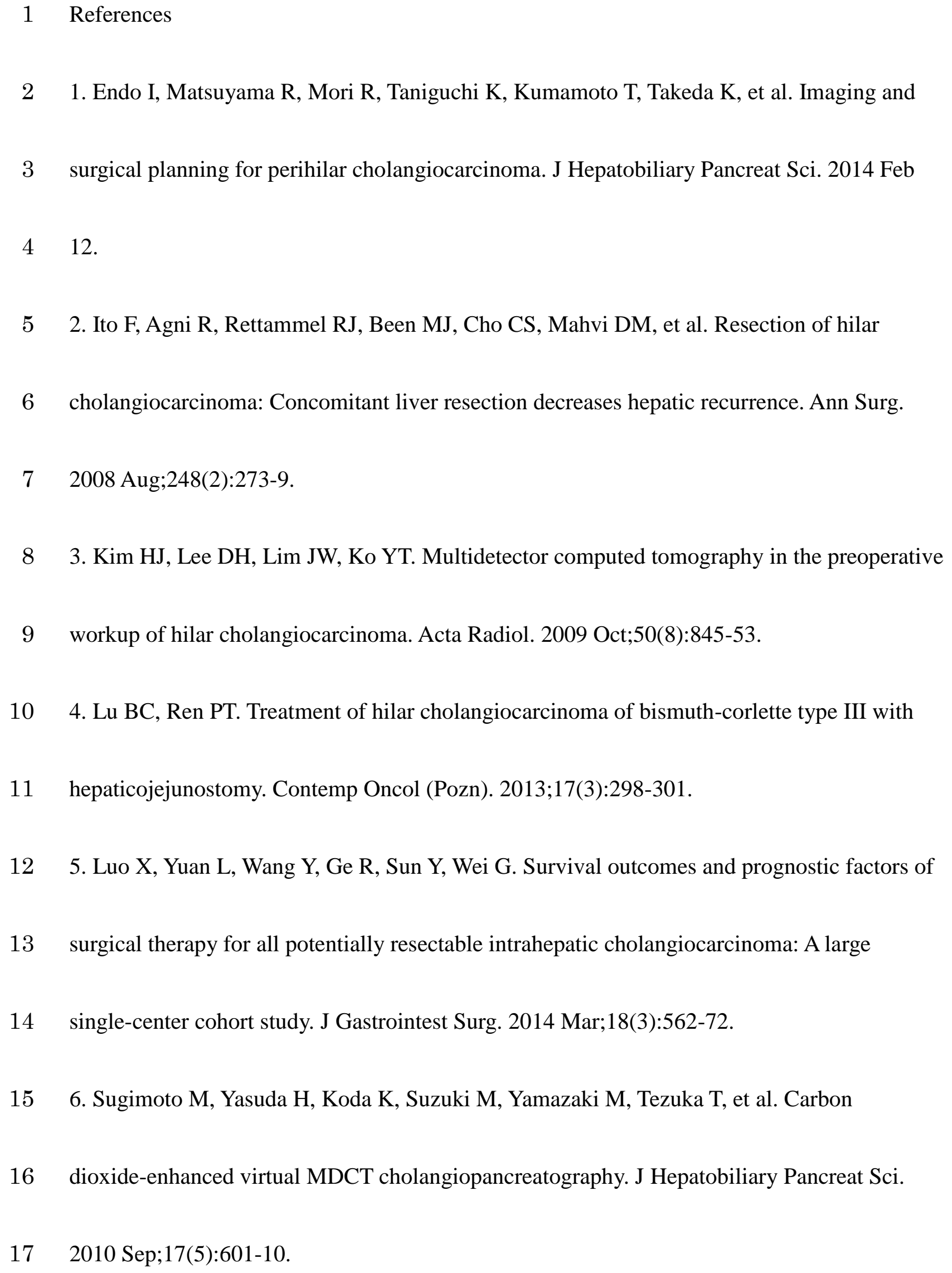




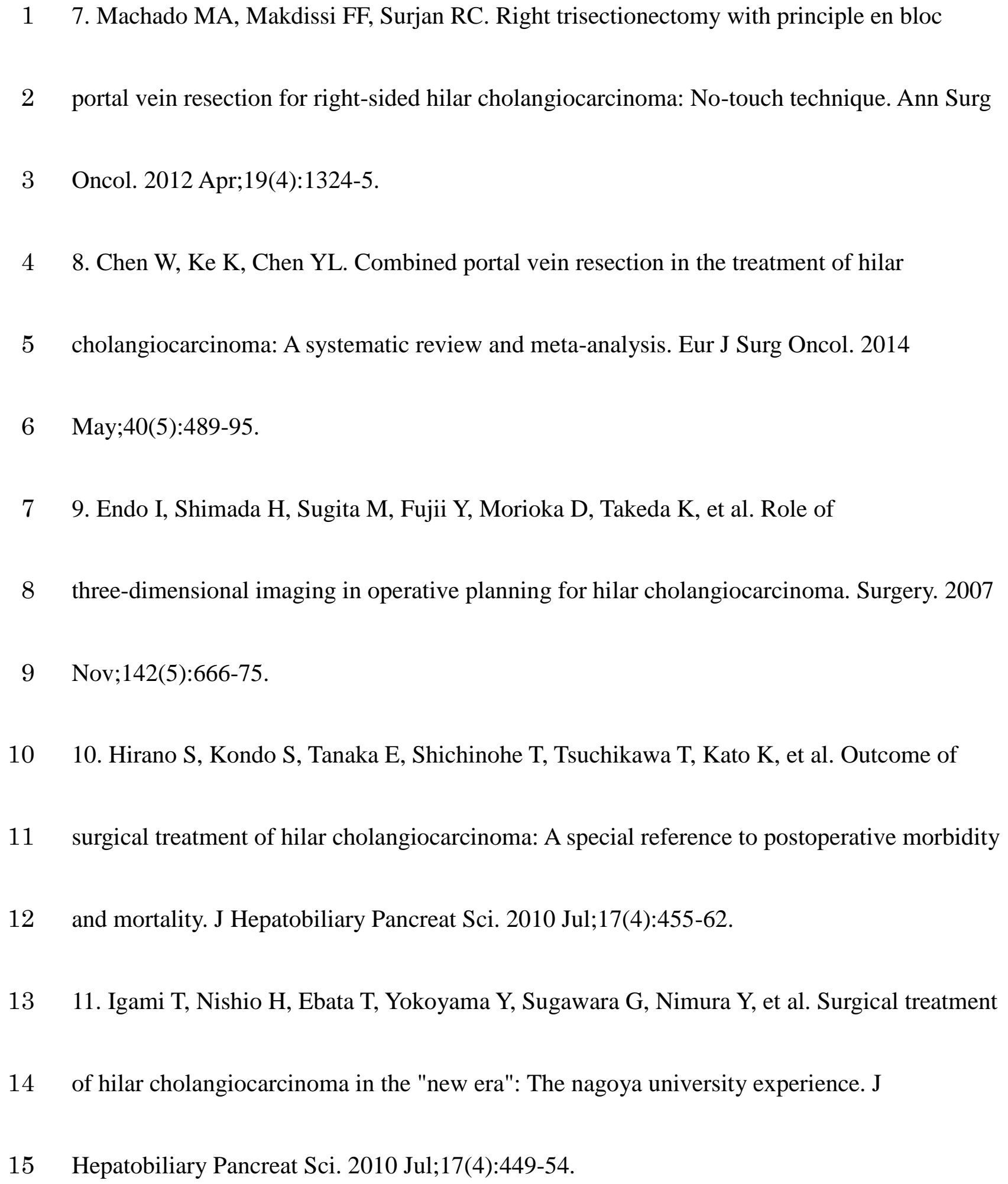


1 12. Matsumoto N, Ebata T, Yokoyama Y, Igami T, Sugawara G, Shimoyama Y, et al. Role of

2 anatomical right hepatic trisectionectomy for perihilar cholangiocarcinoma. Br J Surg. 2014

$3 \quad$ Feb;101(3):261-8.

4 13. Mavros MN, Economopoulos KP, Alexiou VG, Pawlik TM. Treatment and prognosis for

5 patients with intrahepatic cholangiocarcinoma: Systematic review and meta-analysis. JAMA

6 Surg. 2014 Apr 9.

7 14. Miyazaki M, Kimura F, Shimizu H, Yoshidome H, Otuka M, Kato A, et al. One hundred

8 seven consecutive surgical resections for hilar cholangiocarcinoma of bismuth types II, III, IV

9 between 2001 and 2008. J Hepatobiliary Pancreat Sci. 2010 Jul;17(4):470-5.

10 15. Nguyen K, Sing JT,Jr. Review of endoscopic techniques in the diagnosis and management

11 of cholangiocarcinoma. World J Gastroenterol. 2008 May 21;14(19):2995-9.

12 16. Kalra VB, Gilbert JW, Krishnamoorthy S, Cornfeld D. Value of non-contrast sequences in

13 magnetic resonance angiography of hepatic arterial vasculature. Eur J Radiol. 2014

14 Jun;83(6):905-8.

15 17. Rocha FG, Matsuo K, Blumgart LH, Jarnagin WR. Hilar cholangiocarcinoma: The

16 memorial sloan-kettering cancer center experience. J Hepatobiliary Pancreat Sci. 2010

17 Jul;17(4):490-6. 
11 18. Bai Y, Gao F, Gao J, Zou DW, Li ZS. Prophylactic antibiotics cannot prevent endoscopic

3

4

$3 \quad \operatorname{Mar} ; 38(2): 126-30$.

4 19. Zhang R, Zhao L, Liu Z, Wang B, Hui N, Wang X, et al. Effect of CO2 cholangiography

5 on post-ERCP cholangitis in patients with unresectable malignant hilar obstruction - a

6 prospective, randomized controlled study. Scand J Gastroenterol. 2013 Jun;48(6):758-63. 
$1 \quad 1$ Figure legends

3

4

$2 \quad$ Figure 1

3 Summary of the study population in the present study

4 Eighty seven patients with biliary tract malignancy underwent three-dimensional computed

5 tomography (3DCT) cholangiography between October 2009 and September 2014. Of these

6 patients, 21 patients did not undergo laparotomy for the following reasons: 3 patients had

7 distant metastases (liver metastases in 2 patients and supraclavicular lymph node metastases

8 in 1 patient); 10 patients had excessive tumor spread (longitudinal in 6 patients and vertical in

94 patients); 5 patients had insufficient remnant liver volume; 2 patients refused surgical

10 treatment; and 1 patient died of primary disease prior to surgery. Sixty-six patients underwent

11 laparotomy. Resection was abandoned after laparotomy in 10 patients, because of distant

12 metastases in 7 patients (peritoneal dissemination in 4 patients, para-aortic lymph node

13 metastases in 2 patients and liver metastases in 1 patient) and vertical tumor invasion to the

14 duodenum in 3 patients. Fifty-six patients underwent resection with curative intent. Of these

1556 patients, 3 underwent hepatectomy without extrahepatic bile duct resection, and 4

16 underwent pancreaticoduodenectomy (PD) without liver resection. Eventually, forty-nine

17 patients underwent major hepatic resection (MHR) with extrahepatic bile duct resection 
1 (BDR) or MHR with pancreaticoduodenectomy (MHR + PD). Sixty nine patients who

2 underwent MHR + BDR or MHR + PD before the introduction of 3DCT cholangiography

3 were served as the historical control.

4 CCA; Cholangiocarcinoma

6 Figure 2

7 The protocol for 3-dimensional computed tomography cholangiography with carbon dioxide

8 as the contrast medium for the bile duct (a) and with iodine as contrast medium for the bile

$9 \operatorname{duct}(b)$.

10 All imaging was performed using a 64-multi-row detector computed tomography scanner

11 (Aquilion 64, Toshiba Medical Systems, Tochigi, Japan). Scanning parameters were $120 \mathrm{kVp}$,

12 approximately $250-350 \mathrm{mAs}$ adjusted according to patient size (minimum 140 to maximum

$13500 \mathrm{mAs}$ [Sure Exposure, Toshiba Medical Systems]), 0.5-mm section collimation, and a

14 pitch factor of 0.828 during a single breath-hold volumetric acquisition. Images were obtained

15 in a craniocaudal direction and reconstructed with 1-mm slice thickness and 1-mm intervals.

16 Nonionic iodinated contrast material (Iomeron 350; Esai Co, Ltd, Tokyo, Japan) at $2 \mathrm{~mL} / \mathrm{kg}$

17 with a maximum dose of $135 \mathrm{~mL}$ was injected through the antecubital vein at a rate of $4 \mathrm{~mL} / \mathrm{s}$. 


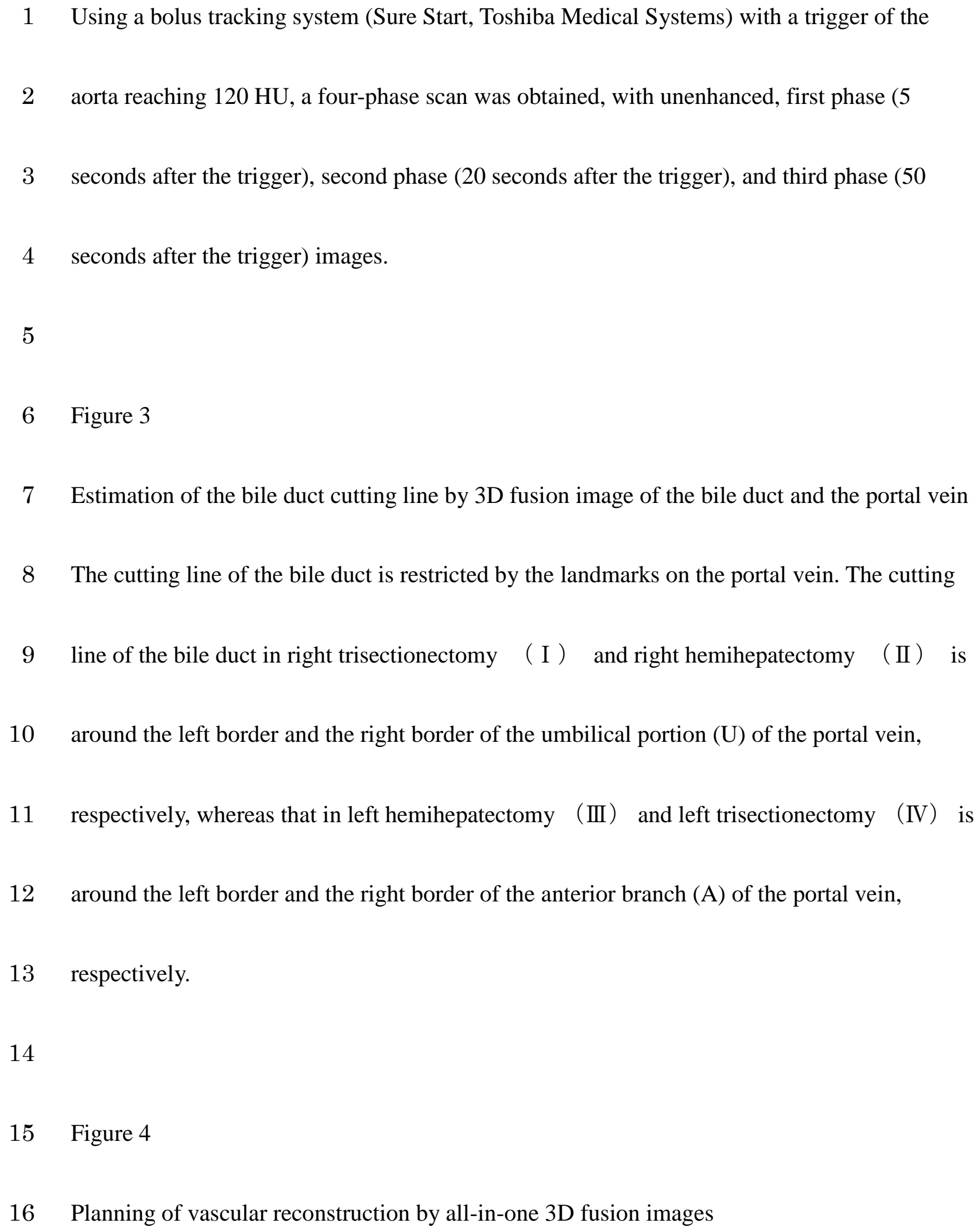

$7 \quad$ Estimation of the bile duct cutting line by 3D fusion image of the bile duct and the portal vein

8 The cutting line of the bile duct is restricted by the landmarks on the portal vein. The cutting

9 line of the bile duct in right trisectionectomy (I ) and right hemihepatectomy (II) is 10 around the left border and the right border of the umbilical portion (U) of the portal vein,

11 respectively, whereas that in left hemihepatectomy (III) and left trisectionectomy (IV) is 12 around the left border and the right border of the anterior branch (A) of the portal vein,

13 respectively.

15 Figure 4

16 Planning of vascular reconstruction by all-in-one 3D fusion images 


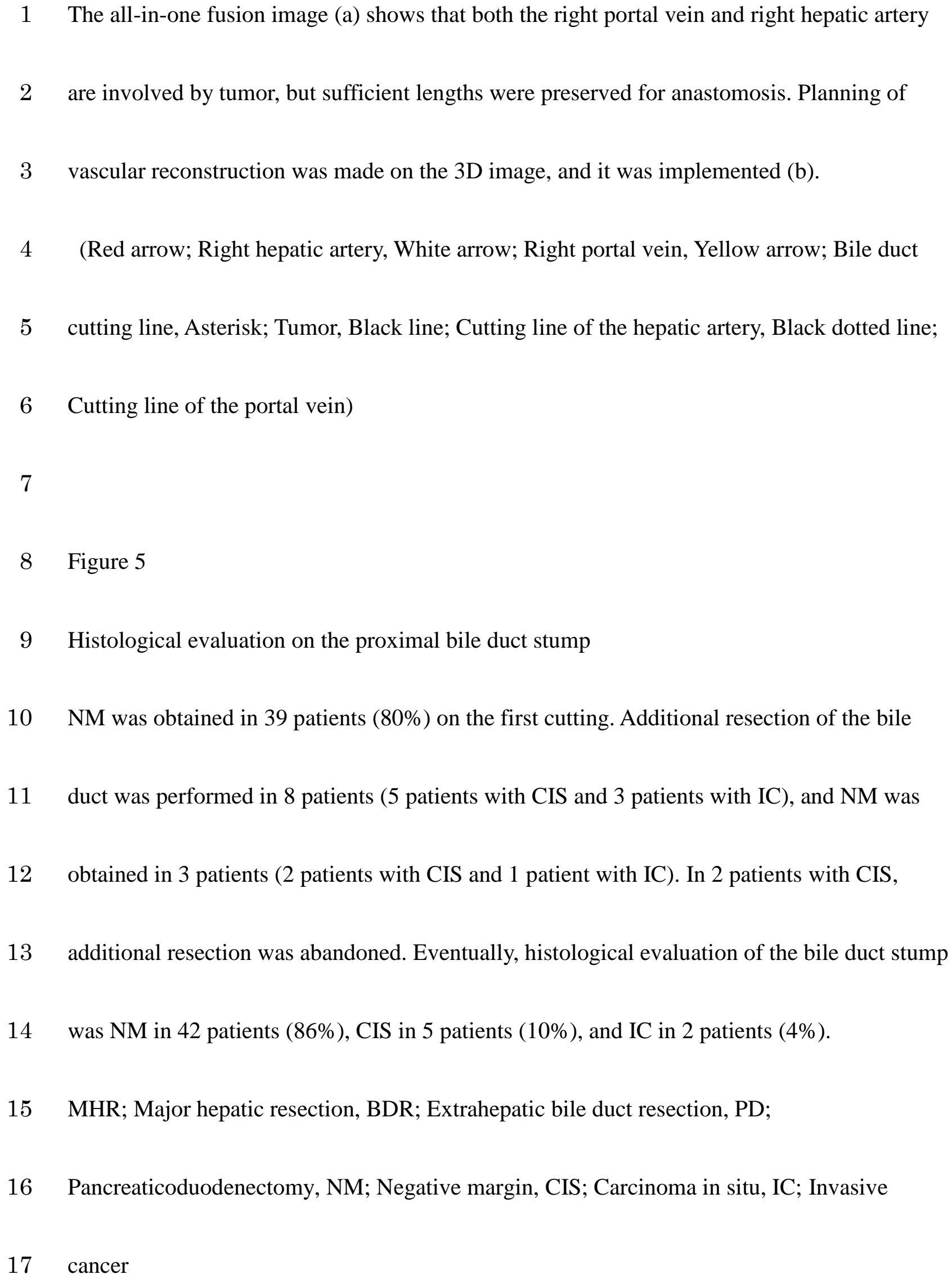




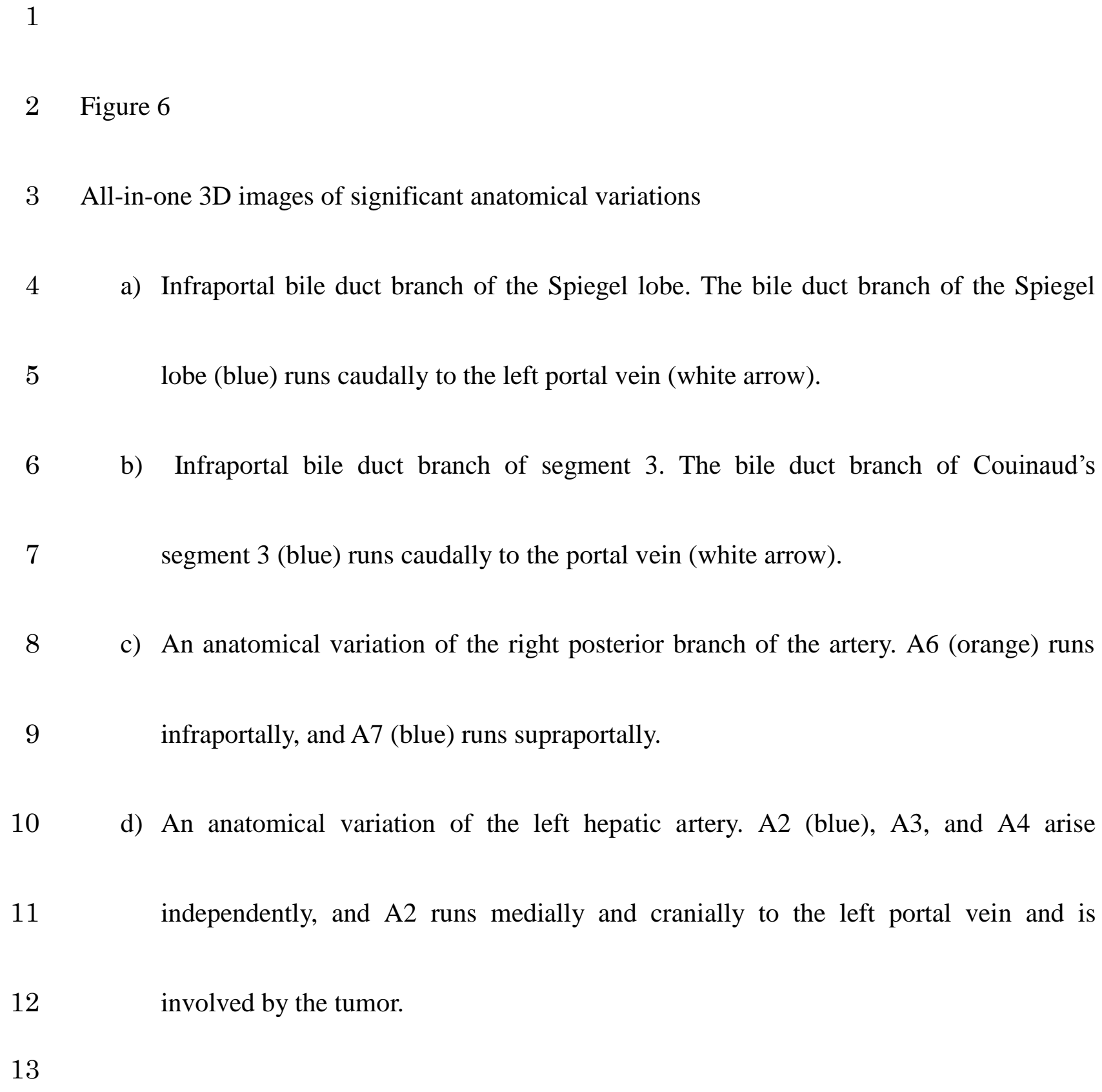

6 b) Infraportal bile duct branch of segment 3. The bile duct branch of Couinaud's

$7 \quad$ segment 3 (blue) runs caudally to the portal vein (white arrow).

8 c) An anatomical variation of the right posterior branch of the artery. A6 (orange) runs

$9 \quad$ infraportally, and A7 (blue) runs supraportally.

10 d) An anatomical variation of the left hepatic artery. A2 (blue), A3, and A4 arise 11 independently, and A2 runs medially and cranially to the left portal vein and is 12 involved by the tumor. 
Table 1

Patients' characteristics; 3DCT cholangiography

\begin{tabular}{lccc} 
& Contrast media & $\mathrm{CO} 2$ & Iodine \\
& $(\mathrm{n}=81)$ & $(\mathrm{n}=15)$ & $P$ value \\
\hline age & $64 \pm 10$ & $64 \pm 15$ & n.s. \\
gender (male/female) & $55 / 26$ & $11 / 4$ & n.s.
\end{tabular}

Location of tumor

$\begin{array}{lcc}\text { Perihilar } & 43 \%(\mathrm{n}=34) & 47 \%(\mathrm{n}=7) \\ \text { Extrahepatic } & 17 \%(\mathrm{n}=14) & 0 \%(\mathrm{n}=0) \\ \text { Intrahepatic } & 27 \%(\mathrm{n}=22) & 47 \%(\mathrm{n}=7) \\ \text { Gallbladder } & 12 \%(\mathrm{n}=10) & 7 \%(\mathrm{n}=1) \\ \text { Intrahepatic and extrahepatic } & 2 \%(\mathrm{n}=1) & \end{array}$

Biliary drainage

$\begin{array}{lcc}\text { ENBD } & 86 \%(\mathrm{n}=70) & 100 \%(\mathrm{n}=15) \\ \text { PTBD } & 7 \%(\mathrm{n}=5) & 0 \%(\mathrm{n}=0) \\ \text { Both } & 6 \%(\mathrm{n}=6) & 0 \%(\mathrm{n}=0)\end{array}$

Maximum order

of the visualized biliary branch

$5(3-6) \quad 5(3-6) \quad$ n.s.

Adverse effect

$\begin{array}{lll}\text { Cholangitis } & 1 \%(\mathrm{n}=1) & 0 \%(\mathrm{n}=0) \\ \text { Elevation of liver enzyme } & 1 \%(\mathrm{n}=1) & 13 \%(\mathrm{n}=2)\end{array}$

Post 3DCT hematological biomarkers

$\begin{array}{lccc}\text { WBC }\left(10^{3} / \mu \mathrm{L}\right) & 5.5 \pm 3.3 & 6.1 \pm 1.9 & \text { n.s. } \\ \text { AST }(\mathrm{IU} / \mathrm{L}) & 60 \pm 46 & 42 \pm 19 & \text { n.s. } \\ \text { ALT }(\mathrm{IU} / \mathrm{L}) & 99 \pm 87 & 53 \pm 25 & \text { n.s. } \\ \text { T.Bil }(\mathrm{mg} / \mathrm{dL}) & 2.0 \pm 2.9 & 1.3 \pm 0.9 & \text { n.s. } \\ \text { LDH (IU/L) } & 190 \pm 72 & 170 \pm 28 & \text { n.s. } \\ \text { ALP }(\mathrm{IU} / \mathrm{L}) & 699 \pm 489 & 802 \pm 436 & \text { n.s. } \\ \gamma \mathrm{GTP}(\mathrm{IU} / \mathrm{L}) & 321 \pm 271 & 371 \pm 191 & \text { n.s. }\end{array}$


1

2

3

4

5

6

7

8

9

10

11

12

13

14

15

16

17

18

19

20

21

22

23

24

25

26

27

28

29

30

31

32

33

34

35

36

37

38

39

40

41

42

43

44

45

46

47

48

49

50

51

52

53

54

55

56

57

58

59

60

61

62

63

64

65

Other preoperative examinations

\begin{tabular}{llll} 
IDUS & $63 \%(\mathrm{n}=51)$ & $47 \%(\mathrm{n}=7)$ & n.s. \\
EUS & $41 \%(\mathrm{n}=33)$ & $20 \%(\mathrm{n}=3)$ & n.s. \\
POCS & $16 \%(\mathrm{n}=13)$ & $27 \%(\mathrm{n}=4)$ & n.s. \\
Tumor biopsy & $72 \%(\mathrm{n}=58)$ & $67 \%(\mathrm{n}=10)$ & n.s. \\
Step biopsy & $57 \%(\mathrm{n}=46)$ & $47 \%(\mathrm{n}=7)$ & n.s. \\
\hline
\end{tabular}

ENBD; Endoscopic nasobiliary drainage,

PTBD; Percutaneous transhepatic biliary drainage,

IDUS; Intraductal ultrasonography,

EUS; Endoscopic ultrasound,

POCS; Peroral cholangioscopy 
Table 2

Comparison with historical control

\begin{tabular}{|c|c|c|c|c|}
\hline & & $\begin{array}{l}\text { 3D group } \\
(\mathrm{n}=49)\end{array}$ & $\begin{array}{l}\text { Historical group } \\
\qquad(\mathrm{n}=69)\end{array}$ & $P$ value \\
\hline age & & $64 \pm 11$ & $66 \pm 9$ & n.s. \\
\hline gender & male/female & $34 / 15$ & $32 / 37$ & 0.01 \\
\hline \multirow[t]{5}{*}{ Location of tumor } & Perihilar & $50 \%(\mathrm{n}=25)$ & $61 \%(n=42)$ & \\
\hline & Extrahepatic & $10 \%(\mathrm{n}=5)$ & $6 \%(n=4)$ & \\
\hline & Intrahepatic & $28 \%(\mathrm{n}=14)$ & $22 \%(\mathrm{n}=15)$ & \\
\hline & Gallbladder & $8 \%(n=4)$ & $12 \%(n=8)$ & \\
\hline & Intrahepatic and extrahepatic & $2 \%(\mathrm{n}=1)$ & & \\
\hline \multirow[t]{2}{*}{ Tumor marker } & CEA & $6.0 \pm 16.5$ & $4.4 \pm 8.7$ & n.s. \\
\hline & CA19-9 & $423 \pm 1476$ & $564 \pm 1708$ & n.s. \\
\hline Subtype of biliary cancer & Papillary type & $16 \%(\mathrm{n}=8)$ & $13 \%(n=9)$ & n.s. \\
\hline \multirow[t]{5}{*}{ Type of surgery } & Right hemihepatectomy & $45 \%(n=22)$ & $33 \%(n=23)$ & n.s. \\
\hline & Left hemihepatectomy & $27 \%(\mathrm{n}=13)$ & $32 \%(\mathrm{n}=22)$ & \\
\hline & Right trisectionectomy & $8 \%(n=4)$ & $20 \%(n=14)$ & \\
\hline & Left trisectionectomy & $20 \%(\mathrm{n}=10)$ & $14 \%(n=10)$ & \\
\hline & $\mathrm{MHR}+\mathrm{PD}$ & $24 \%(\mathrm{n}=12)$ & $10 \%(\mathrm{n}=7)$ & 0.04 \\
\hline \multirow[t]{2}{*}{ Vascular reconstruction } & Portal vein & $39 \%(\mathrm{n}=19)$ & $10 \%(\mathrm{n}=7)$ & 0.0002 \\
\hline & Hepatic artery & $14 \%(\mathrm{n}=7)$ & $10 \%(n=7)$ & n.s. \\
\hline Blood loss & & $2687 \pm 3685$ & $1750 \pm 1609$ & n.s. \\
\hline Operative time & & $782 \pm 277$ & $635 \pm 123$ & 0.0002 \\
\hline \multirow[t]{4}{*}{ TNM stage } & $\mathrm{T} 4$ & $43 \%(n=21)$ & $17 \%(\mathrm{n}=12)$ & 0.0025 \\
\hline & N1 & $43 \%(n=21)$ & $48 \%(n=33)$ & n.s. \\
\hline & M & $0 \%(\mathrm{n}=0)$ & $10 \%(n=7)$ & 0.0052 \\
\hline & StageIV & $41 \%(n=20)$ & $35 \%(n=24)$ & n.s. \\
\hline
\end{tabular}


1

Bile duct stump

First cutting

Additional resection

Surgical radicality
Negative margin

Carcinoma in situ

Invasive cancer

Invasive cancer free ratio

Negative margin
Carcinoma in situ
Invasive cancer

Negative margin
Carcinoma in situ
Invasive cancer

Negative margin
Carcinoma in situ
Invasive cancer

Invasive cancer free ratio

R0

$$
\begin{array}{cc}
80 \%(n=39) & 74 \%(n=51) \\
14 \%(n=7) & 6 \%(n=4) \\
6 \%(n=3) & 20 \%(n=14)
\end{array}
$$

35

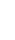<smiles>C1CCC1</smiles> 
(Jan.2004-Aug. 2009)

$$
\text { MHR + BDR }
$$

or

MHR + PD

69 patients
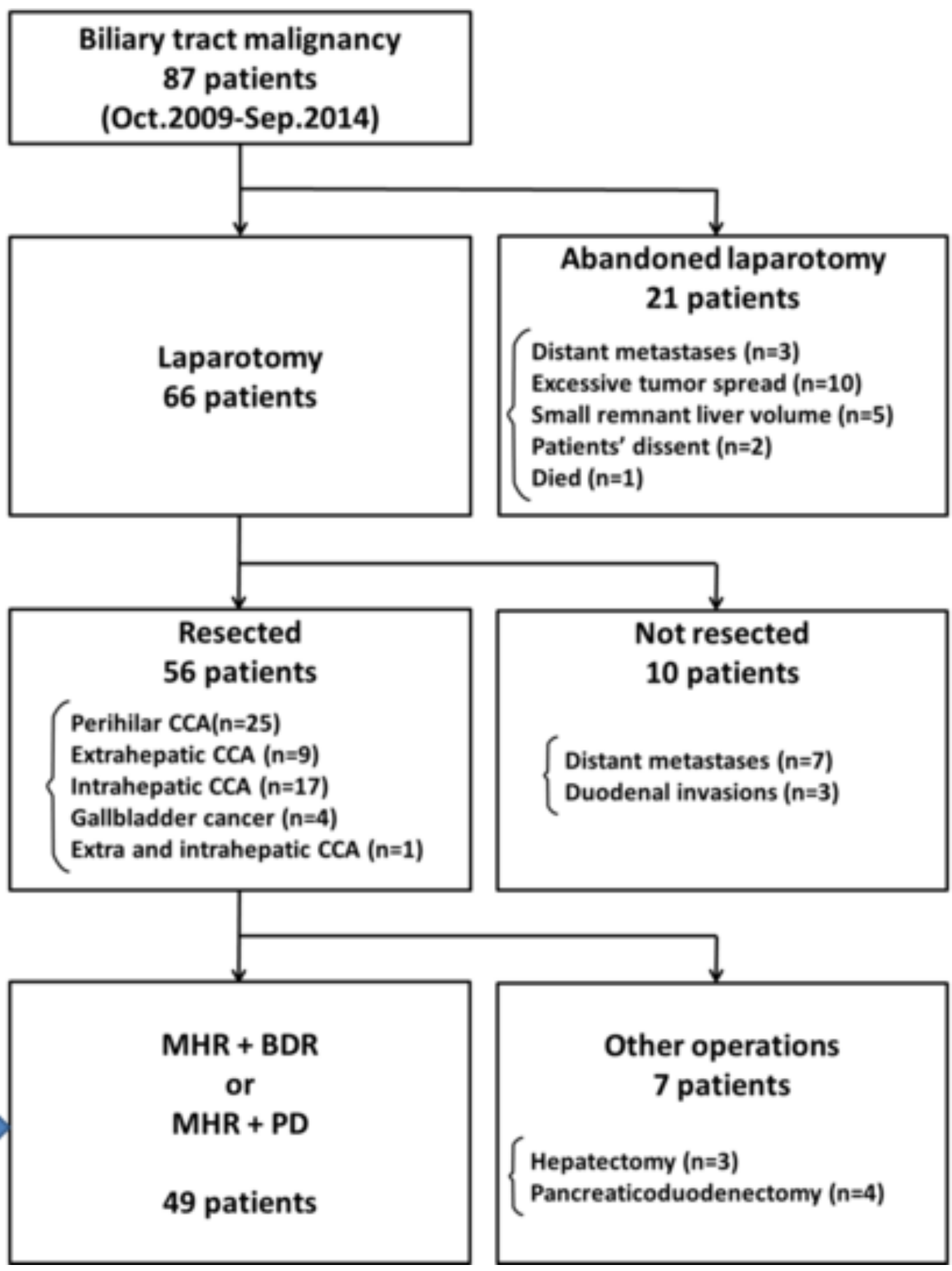
Click here to download high resolution image

a) $\mathrm{CO}_{2}$ cholangiography $(\mathrm{N}=81)$

(1) $\mathrm{CO}_{2}$ injection $(10-120 \mathrm{ml})$<smiles>C[14CH3]</smiles>

(2) Precontrast scan<smiles>[3H][Tl]</smiles>

(3) Intravenous injection of contrast material Additional injection of $\mathrm{CO}_{2}$

(4) Arterial phase Extraction of the bile duct and the artery

(5) Portal phase Extraction of the portal vein

b) Iodine cholangiography ( $N=15$ )

(1) Intravenous injection of contrast material

(2) Arterial phase

Extraction of the artery

(3) Portal phase

Extraction of the portal vein

$\downarrow$

(4) Diluted iodine injection<smiles>C[Tl]</smiles>

(5) Biliary phase 
Click here to download high resolution image

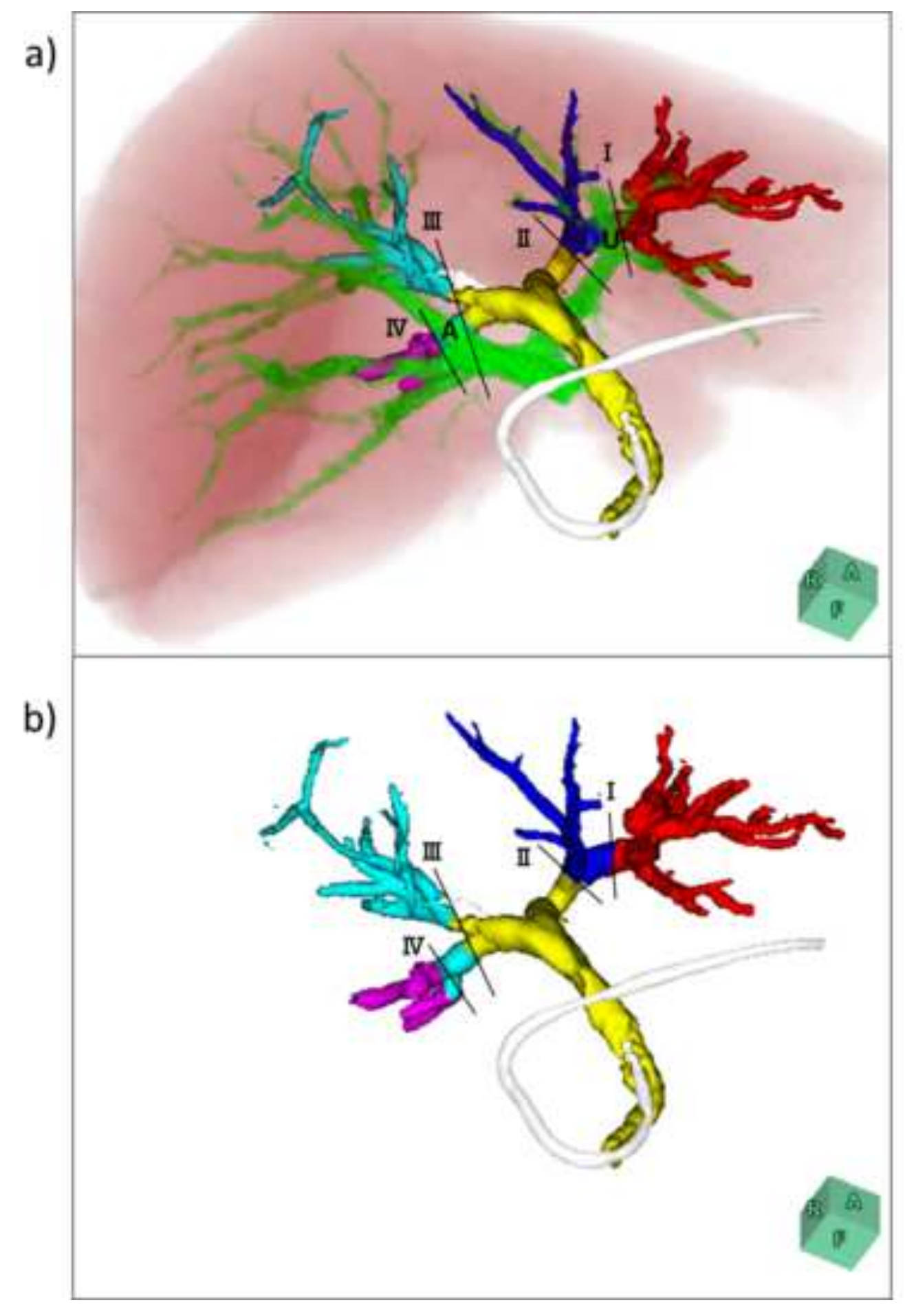


Click here to download high resolution image

a)

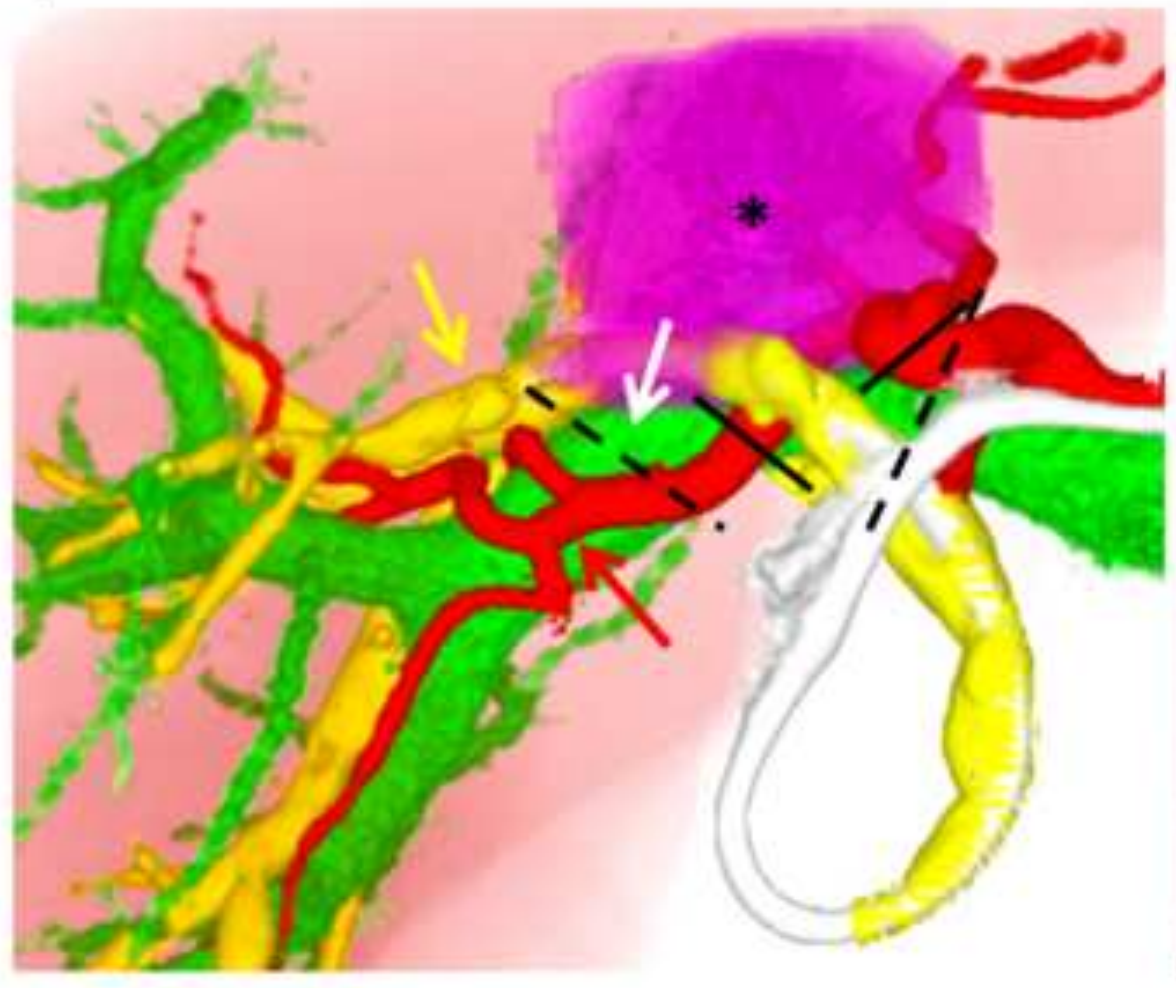

b)

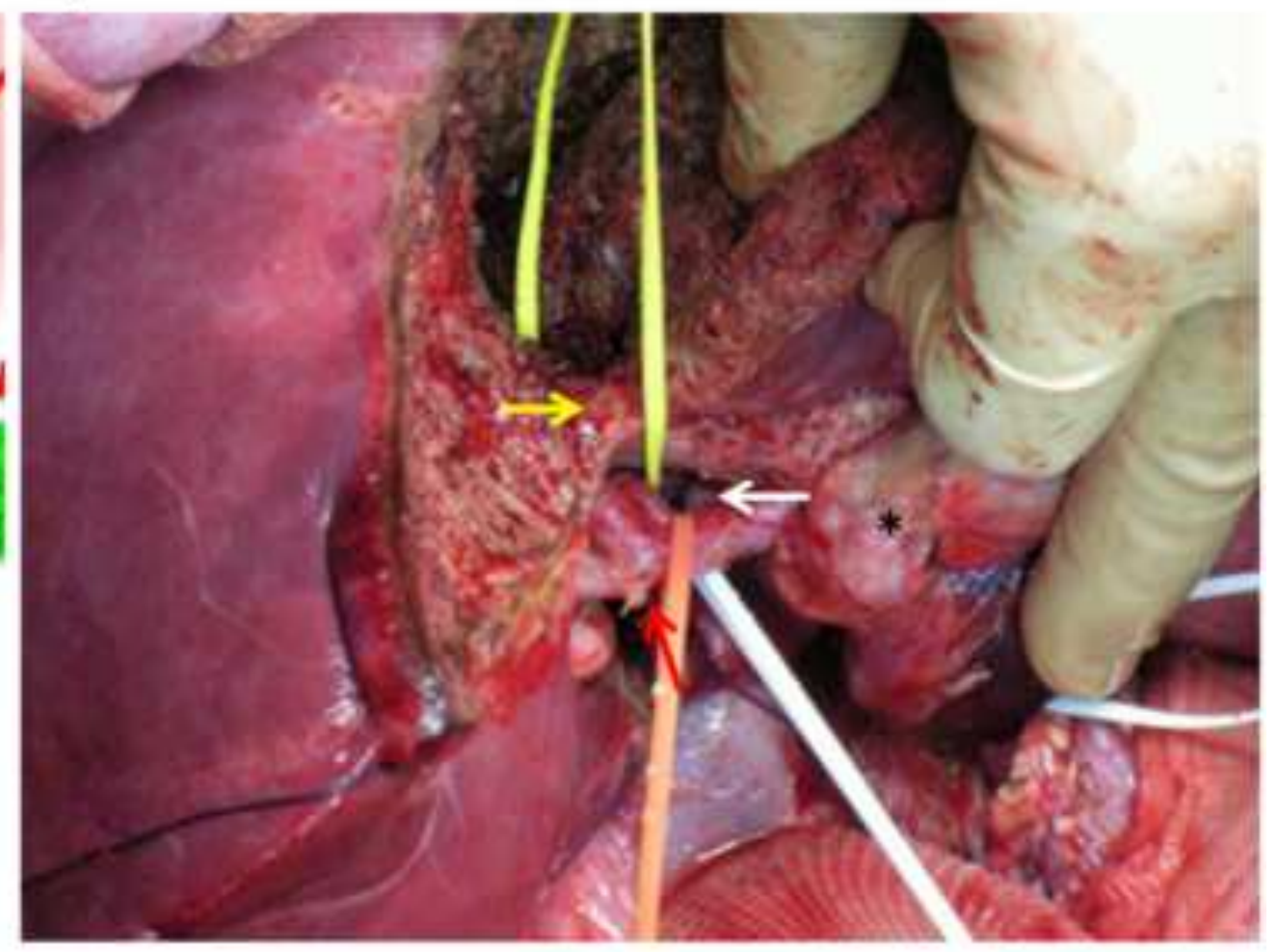




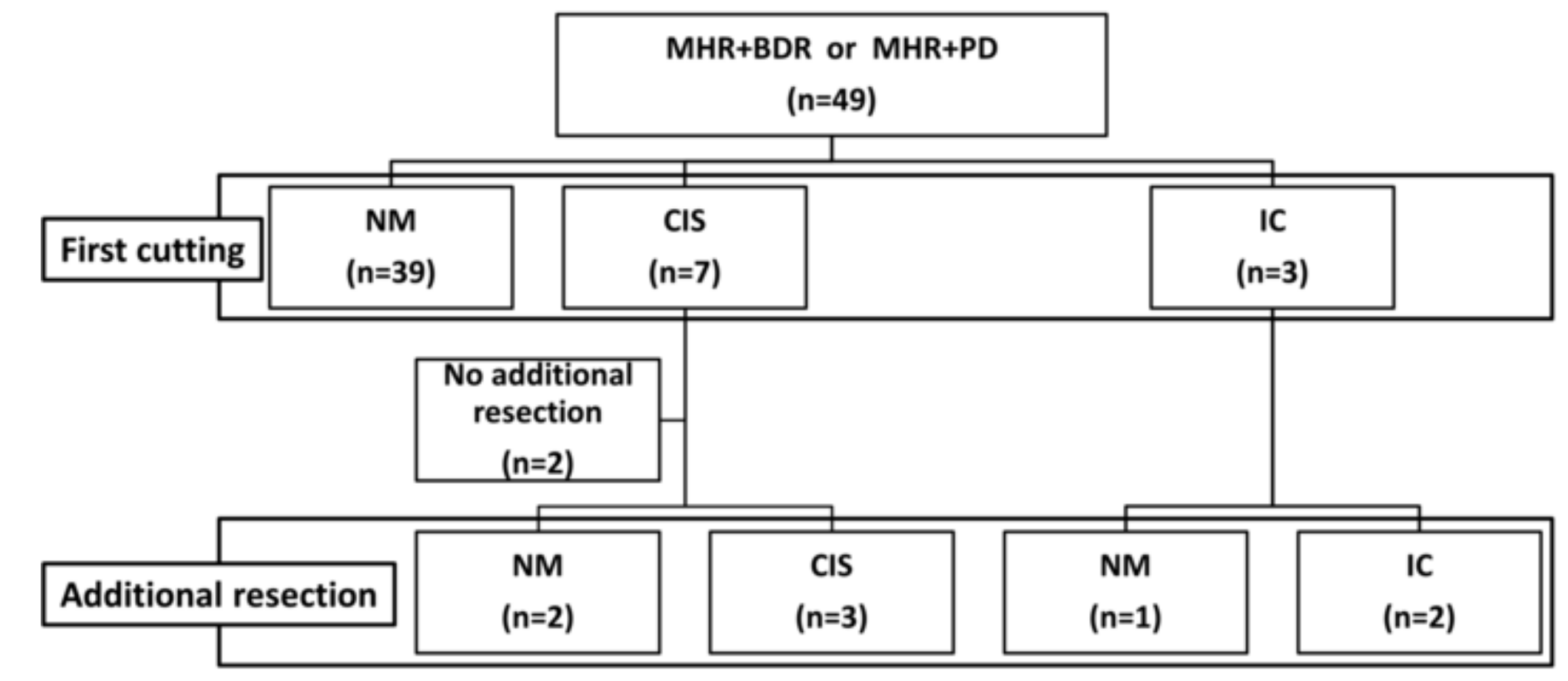


Click here to download high resolution image

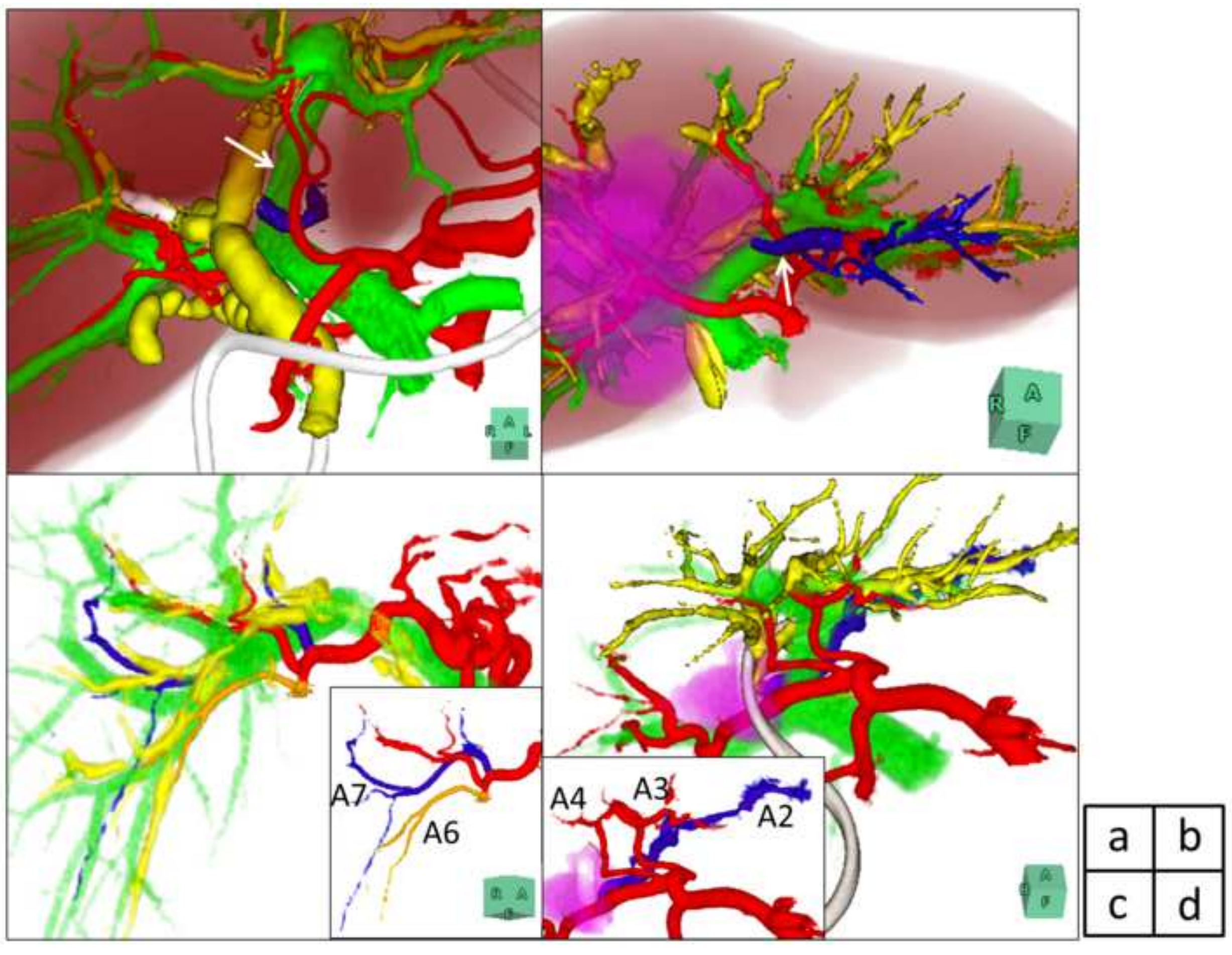




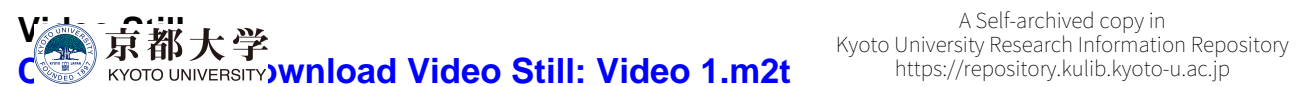

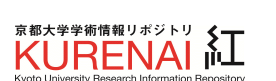


V: 京都大学 


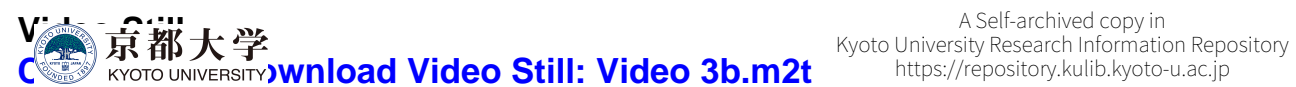

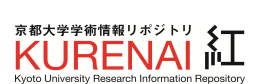




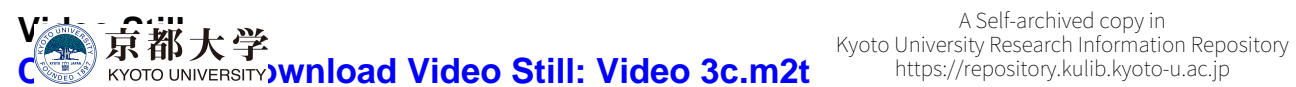

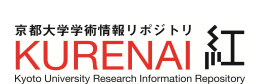

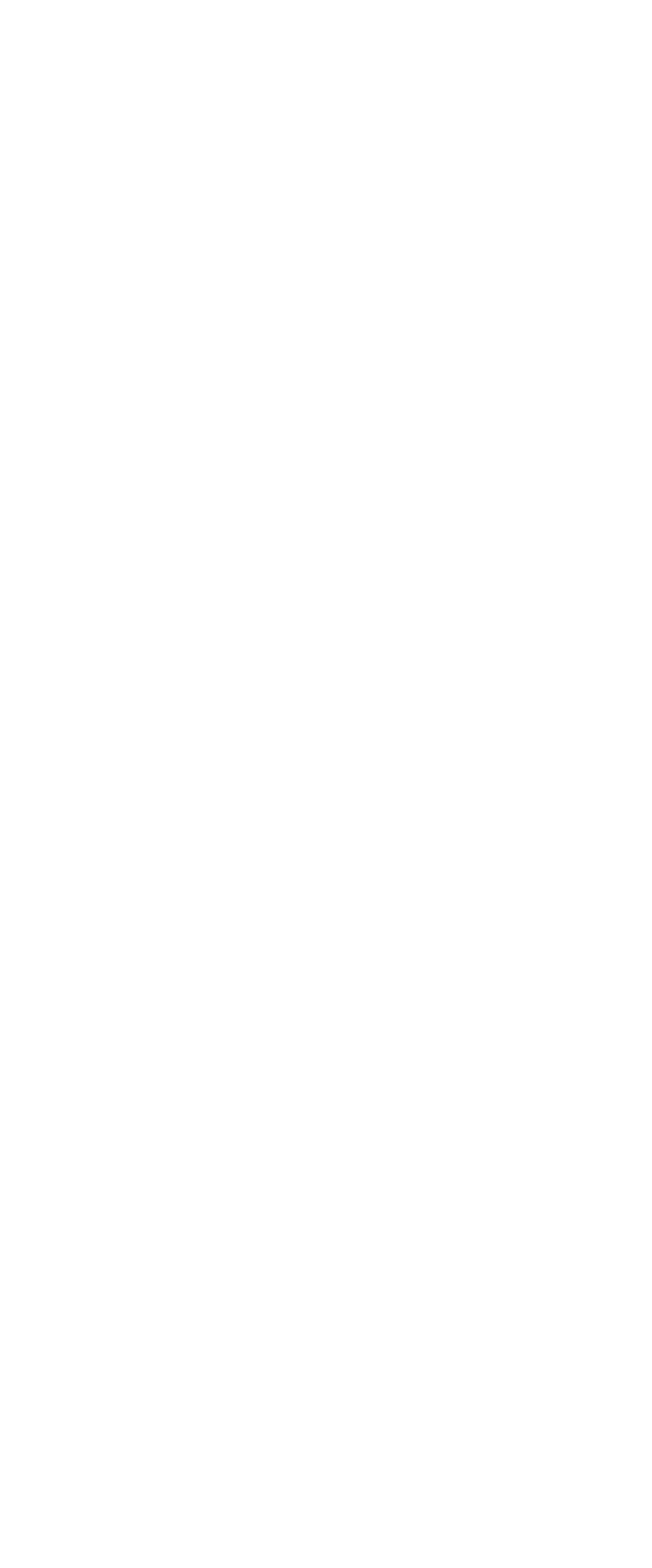




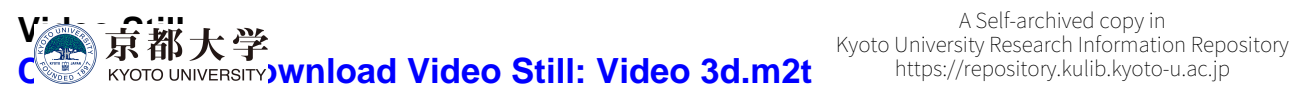

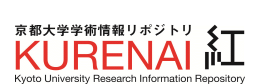

